L'H. triquetrum (L.) est commun aux environs de Montluçon, mais fructifie assez rarement. Je ne l'ai rencontré en fruit que dans la vallée de l'Amaron, au Roc-du-Saint, en remontant le ruisseau de Marignon, qui aboutit entre le prenier et le deuxième tunnel du chemin de fer de Moulins. Les $H$. splendens et squarrosum sont généralement stériles.

\title{
SÉANCE DU 24 NOVEMBRE 1871.
}

PRÉSIDENCE DE M. GERMAIN DE SAINT-PIERRE.

M. Larcher, vice-secrétaire, donne lecture du procès-verbal de la séance du 10 novembre, dont la rédaction est adoptée.

M. le Président prononce l'allocution suivante :

Messieurs,

Depuis le jour où je vous adressais l'expression de mes profonds regrets, de n'avoir pu, pendant l'investissement de Paris par les armées étrangères, venir vous rejoindre ici et partager avec vous les diverses épreuves de ces temps si douloureux, des journées plus néfastes encore, de plus cruels désastres ont frappé au cœur notre cher Paris. - Paris, ce grand navire alors désemparé, Paris, ce gigantesque radeau de la Méduse, a vu porter des torches incendiaires au milieu des trésors de la science et de l'art que l'ouragan d'obus et de mitraille avait épargnés.

$\Delta$ ujourd'hui, Messieurs, je suis heureux qu'il me soit donné, au nom de la Société botanique de France, de remercier cordialement ceux d'entre vous qui, avec une si louable fermeté, ont occupé ici un poste d'honneur, pendant la longue durée de ces temps si lamentables, et qui, en continuant à se grouper, calmes au milieu de la tourmente, sans se laisser aller au découragement, sans consulter le danger, ont protesté par leur présence contre une seconde invasion des barbares!

Le compte rendu de ces simples et pourtant solennelles séances vous dira ceux que la tempête n'a pu disperser qu'au dernier jour.

Pendant ces temps si difficiles, plusieurs d'entre vous, Messieurs, et notamment MM. Ernest Roze, Maxime Cornu, Cauvet, ont fait preuve d'un zèle scientifique que ne saurait jamais oublier notre Société, en alimentant d'articles pleins d'intérêt notre Bulletin, dont les pages sans eux fussent restées désertes. J'ai lu avec un plaisir tout particulier les savantes observations et les notes critiques dans lesquelles M. Cauvet a passé en revue quelques-uns des sujets favoris de mes études, et j'éprouverai une satisfaction infinie à répondre à mon habile contradicteur. 
Au nom de la Société botanique, j'adresse tout spécialement de chaleureux remercîments à M. Ernest Roze, l'un de nos honorables vice-présidents, qui, pendant mon involontaire absence, a présidé sans interruption nos séances, depuis le mois de novembre 1870 jusqu'au mois de juin 1871; - à MM. Larcher et Delondre, qui ont contribué aux fonctions du secrétariat avec un infatigable dévonement ; - et à $M$. de Schœnefeld, notre digne secrétaire général, qui, malgré sa santé altérée par de dures privations, n’a pas cessé un seul instant, pendant les deux siéges de Paris, de se dévouer à ses laborieuses et difficiles fonctions; et qui, pilote habile, a pu, à travers mille obstacles et en dépit de ces temps néfastes, fairc parvenir à bon port les deux dernières années de notre Bulletin et le compte rendu de notre session d'Autun-Givry, cette session si joyeuse et si bien remplie qui précédait de si peu les calamités et les désastres de la guerre. Vous le savez, Messieurs, au moment où Paris se trouvait presque désert, et où ses rares habitants étaient encore terrifiés par les événements inouïs dont ils venaient d'être témoins, $\mathbf{M}$. de Schœnefeld se rendait ici à son poste, le 26 mai, à l'heure fixée pour la séance, et y représentait seul la Société, dont la courageuse persévérance et la ferme attitude s'étaient solidement maintenues jusqu'à l'explosion suprême de l'ouragan le plus antipatriotique et le plus antisocial dont les générations conserveront le souvenir.

J'ajoute en terminant ce rapide tableau : La Société boranique de France,

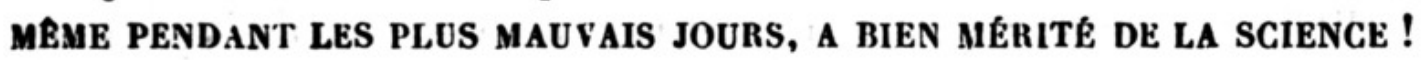

Messieurs, durant les dernières vacances, la Société botanique a fait des pertes profondément regrettables en la personne de plusieurs de ses membres éminents. Notre savant coufrère $M$. Henri Lecoq (de clermont-Ferrand), naguère encore plein de force et de santé, a succombé en quelques jours à une maladie aiguë, dans la force de l'àge et laissant d'importants travaux inachevés. M. H. Lecoq unissait aux aptitudes du vrai naturaliste, à la sagacité de l'observateur, les brillantes facultés de l'écrivain, la finesse et la malicieuse gaieté du conteur, et les solides qualités du cœur. La perte que les amis des sciences naturelles font en la personne de $M$. H. Lecoq ne sera pas moins sensible aux géologues qu'aux botanistes. Parmi tant de travaux estimables dus à la plume facile de $\mathrm{M}$. H. Lecoq, je rappellerai surtout l'important ouvrage (9 volumes in-octavo) intitulé : Études sur la géographie botanique de l'Europe, et en particulierdu plateau central de la France. Vulgarisateur infatigable des sciences qu'il cultivait, M. H. Lecoq publiait, il y a deux ans à peine, son livre ingénieux et élégant intitulé : le Monde des fleurs. $\mathbf{1}$. Lecoq a légué, nous ai-on dit, à la ville de Clermont-Ferrand ses importantes collections botaniques, zoologiques et minéralogiques (véritable musée des productions naturelles de la France centrale), dont la valeur est considérable. Une notice sur la vie et les travaux de $\mathbf{~ 1 .}$. H. Lecơ, insérée dans le Bulletin de la Société botanique, sera un juste hommage rendı à la mémoire de l'éminent naturaliste. 
Nous apprenons également avec un profond regret la mort de M. Pietro Savi, le savant professeur et directeur du jardin botanique de Pise ; -la mort de M. le docteur Rambur (de Genève), connu des naturalistes surtout par ses importants travaux sur l'entomologie; - la mort de M. l'abbé Jacquel, curé de Coinches (Vosges), dont les recherches, en la savante compagnie de NM. Mougeot, Godron, Kirschleger, etc., ont contribué à compléter les études sur la flore vogéso-rhénane; - et enfin, la perte si prématurée et si regrettable de M. Armand Peyre (de Toulouse), enlevé à l'âge de trente ans, par une maladie rapide, à ses amis et aux recherches qu'il poursuivait avec autant de zèle que de succès.

M. A. de Bouis demande la parole et s'exprime en ces termes :

Aux pertes nombreuses que M. le Président vient d'annoncer à la Société, je crois qu'il serait convenable d'ajouter le nom de Madame veuve Ricard, qui, en consentant à se faire inscrire parmi les membres de la Société, a donné un bon exemple aux personnes de son sexe (voyez le Bulletin, t. VII, p. 440). Guidée dans ses premières études par l'abbé Le TurquierDelongchamp (auteur de la Flore de Rouen), par son frère M. Arsène Maille (si connu des entomologistes) et par quelques amis, elle trouva un charme dans une science qui ne lui permettait pas de faire un pas sur la terre sans y trouver des problèmes à résoudre. C'est ainsi qu'elle acquit, par une longue et minutieuse application à la détermination des espèces, une connaissance assez sûre pour pouvoir en ajouter quelques-unes à la flore française. Il nous suffira de signaler le Dracocephalum Ruyschiana. Je ne voudrais pas me permettre de faire un éloge dont sa modestie, même après la mort, serait blessée ; car si elle cultivait la botanique avec amour, si elle trouvait les nobles plaisirs de l'intelligence dans cette contemplation des merveilles de la nature, elle évitait avec un soin particulier tout ce qui aurait pu faire croire qu'elle fût savante : contente, jusqu'à la fin de sa vie, de récolter pour son herbier aujourd'hui assez complet des plantes de France, toutes les plantes qu'elle pouvait trouver dans ses nombreux et fréquents voyages dans notre patric. Bienveillante envers tous et surtout envers les botanistes, qui s'empressaient de lui envoyer les plantes qu'elle n'avait pu trouver elle-mème, elle a pu arriver à former une riche collection des plantes françaises, pour laquelle elle a eu comme collaborateurs Requien, J.-B. Mongeot, Maire, Alphonse Maille (son neveu), Aug. Le Prévost, etc. Son savoir était le moindre de ses mérites, et aujourd'hui, dans un monde meilleur, elle jouit des nombreux bienfaits qu'elle a répandus autour d'elle pendant une longue vie, car sa charité était grande, généreuse, ingénieuse, inépuisable, et elle a vécu près d'un siècle.

Par suite des présentations faites dans la dernière séance, M. Je Président proclame l'admission de: 
MM. Franco (Louis), médecin à Machecoul (Loire-Inférieure), présenté par MM. Gobert et Viaud-Grand-Marais;

Posada-Arango (Andres), docleur en médecine, à Médellin (États-Unis de Colombie), présenté par MM. G. Planchon et Bureau.

M. le Président annonce en outre deux nouvelles présentations.

M. Van Tieghem fait à la Société la communication suivante :

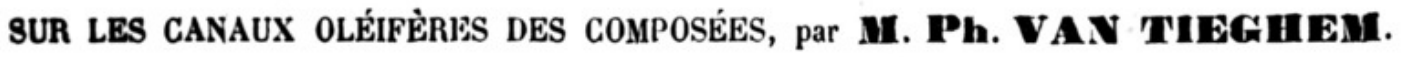

Les plantes de la famille des Composées forment dans la profondeur des tissus de leurs divers organes des huiles essentielles incolores ou diversement colorées, dont quelques-unes ont fait l'objet d'études chimiques intéressantes.

On sait que ces huiles essentielles sont des mélanges d'un hydrocarbure liquide de la forme $\mathrm{C}^{\mathrm{m}} \mathrm{H}^{\mathrm{n}}$, ordinairement isomère de l'essence de térébenthine $\mathrm{C}^{20} \mathrm{H}^{16}$, et d'une essence oxygénée solide et cristallisable de la forme $\mathrm{C}^{\mathrm{m}} \mathrm{H}^{\mathrm{n}} \mathrm{O}^{2}$, le plus souvent isomère du camphre du Japon $\mathrm{C}^{20} \mathrm{H}^{16} \mathrm{O}^{2}$. Cette essence oxygénéc est tenue en dissolution par l'hydrocarbure dont elle paraît dériver par simple oxydation. Ainsi, pour en citer quelques exemples, l'huile essentielle de Matricaire (Matricaria Parthenium L.) est un mélange d'un hydrogène caṛboné et d'une essence oxygénée solide qui présente la même composition que le camphre des Laurinées $\mathrm{G}^{20} \mathrm{H}^{16} \mathrm{O}^{2}$, mais qui dévie à gauche le plan de polarisation de la lumière incidente, tandis que le camphre des Laurinées le dévie a droite. L'essence d'Absinthe (Artemisia Absinthium) plusieurs fois rectifiée offre la même composition que le camphre des Laurinées, mais comme lui elle dévie à droite. L'essence de Camomille (Matricaria Chamomilla), qui est bleu d'azur, se solidifie en partie par le froid, et les lamelles cristallines qui s'y déposent sont isomères du camphre du Japon. L'essence de Tanaisie(Tanacetum vulgare) traitée par l'acide chromique produit une substance identique au camphre des Laurinées. L'essence de Camomille romaine (Anthemis nobilis) est un mélange d'un hydrogène carboné $\mathrm{C}^{20} \mathrm{H}^{16}$ isomère de l'essence de térébenthine et d'une huile essentielle oxygénée $\mathrm{C}^{10} \mathrm{H}^{8} \mathrm{O}^{2}$, qui, traitée par la potasse, se convertit en acide angélique. L'essence d'Osmitopsis asteriscoides a la même composition que le camphre de Bornéo $\mathrm{G}^{20} \mathrm{H}^{18} \mathrm{O}^{2}$. La racine d'Aunée (Inula Helenium) contient dans son essence un principe cristallisable odorant, l'hélénine de Gerhardt $\mathrm{C}^{15} \mathrm{H}^{10} \mathrm{O}^{2}$, d'où l'on extrait par élimination de deux équivalents d'eau, l'hélénène $\mathrm{C}^{15} \mathrm{H}^{8}$. Enfin l'essence d'Artemisia contra offre la composition $\mathrm{C}^{24} \mathrm{H}^{20} \mathrm{O}^{2}$, et par distillation sur l'acide phosphorique anhydre elle reproduit le cymène $\mathrm{C}^{24} \mathrm{H}^{18}$. Telle est d'une façon générale la nature ou la qualité de ces huiles essentielles.

Si maintenant, pour se faire une idée de leur quantité, c'est-à-dire de la 


\section{$2 \mathrm{BHL}$ Biodiversity Heritage Library}

1871. "Séance Du 24 Novembre 1871." Bulletin de la Société botanique de France 18, 283-286. https://doi.org/10.1080/00378941.1871.10825366.

View This Item Online: https://www.biodiversitylibrary.org/item/8641

DOI: https://doi.org/10.1080/00378941.1871.10825366

Permalink: https://www.biodiversitylibrary.org/partpdf/159826

\section{Holding Institution}

Missouri Botanical Garden, Peter H. Raven Library

\section{Sponsored by}

Missouri Botanical Garden

\section{Copyright \& Reuse}

Copyright Status: Public domain. The BHL considers that this work is no longer under copyright protection.

This document was created from content at the Biodiversity Heritage Library, the world's largest open access digital library for biodiversity literature and archives. Visit BHL at https://www.biodiversitylibrary.org. 